\title{
Scheduling and Dropping Policies in Delay Tolerant Network
}

\author{
Ruchira more $^{1}$, Milind Penurkar ${ }^{2}$ \\ Student, IT, MIT College of Engineering, Pune, India ${ }^{1}$ \\ Assistant professor, IT, MIT College of Engineering, Pune, India ${ }^{2}$
}

\begin{abstract}
Sometimes end to end connectivity may not exist between the nodes, sometimes it is too expensive to connect all the networks and sometimes due to some catastrophic effect, exchanging of messaging are difficult, so it can cause delays from hours to days, hence networking of this type of situation is called as Delay Tolerant Network. As no end to end connectivity between the nodes so it require buffer at each node to store incoming messages. The network uses a store-carry-forward mechanism, while sending a message from one node to another node. Messages which are stored at each node carry along with him until it reaches to the next node and then forward. Here two scheduling policies are named as PRIORITY+ algorithm and FACTOR algorithm. PRIORITY+ algorithm considers a priority of message with different routing algorithm. FACTOR algorithm considers the TTL value with priority of that particular message. Algorithms can achieve better delivery ratios with less overhead and latency ratio.
\end{abstract}

Keywords: Include at least 4 keywords or phrases.

\section{INTRODUCTION}

A delay tolerant network is a wireless network where node hop by hop. This mechanism is called "store-carrynodes are mobile but there is no end-to-end connectivity forward" mechanism. This type of architecture is called as between nodes [1] [3]. This type of communication Delay Tolerant Network Architecture, shown in Fig.1. In environments subject to delay and disruptions. The order to increase delivery probability we propagated difference between traditional network and delay tolerant multiple replicas of messages [2]. network is shown in table 1 .

\begin{tabular}{|l|l|l|}
\hline & $\begin{array}{l}\text { Traditional } \\
\text { network }\end{array}$ & $\begin{array}{l}\text { Delay Tolerant } \\
\text { network }\end{array}$ \\
\hline $\begin{array}{l}\text { E2E } \\
\text { connectivity }\end{array}$ & Continues & $\begin{array}{l}\text { Frequent } \\
\text { disconnection }\end{array}$ \\
\hline $\begin{array}{l}\text { Propagation } \\
\text { Delay }\end{array}$ & Short & Long \\
\hline $\begin{array}{l}\text { Transmission } \\
\text { reliability }\end{array}$ & High & Low \\
\hline Link data rate & Symmetric & Asymmetric \\
\hline
\end{tabular}

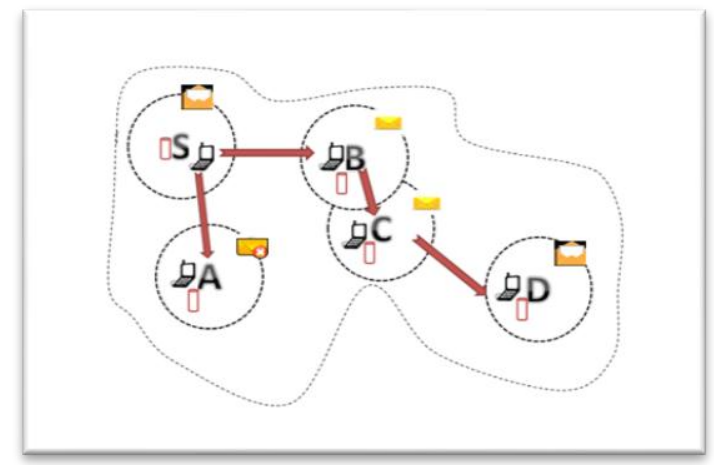

Fig 1: General DTN architecture

When source node is in contact with any intermediate node (the node which is close to destination node) then message(s) stored at source node is forwarded to that node and carry along it till it reaches to the destination node. This process is continued until it reaches to the destination
Delay Tolerant Networking Research group (DTNRG) [4] is very useful while understanding DTN related standards. In DTN architecture, we have a bundle layer. Bundle layer is between the transport layer and application layer. The bundle is nothing but messages or we can say that collection of messages. For keeping a collection at each node we require buffer with efficient management technique which are scheduling policies and dropping policies shown in fig.2. Efficient scheduling decides which messages should be sent first and efficient drop policies will decide which message should be discarded first.

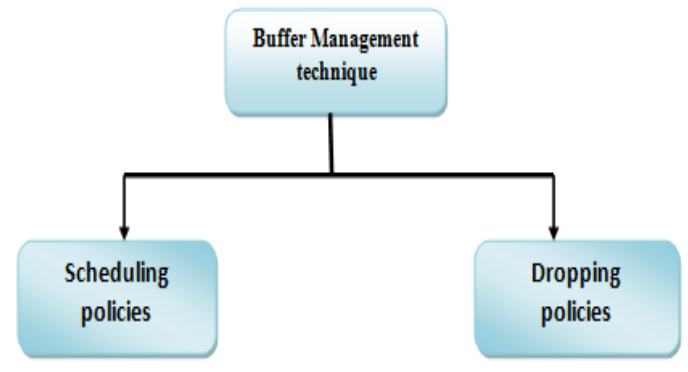

Fig.2.Buffer Management Technique

In this Paper, a Delay Tolerant Network is used to analyze different scheduling policy on different scenarios and introduces a new scheduling policy which gives a better result than already implemented scheduling policy. All the work is done on ONE(opportunistic Network Environment) simulator.

The remainder of the paper is organized as follows. Section II elaborates on the background about scheduling 
and dropping policies for DTNs. Section III presents our DTN laboratory tested, while Section IV focuses on the performance analysis of the proposed approaches. Finally, Section V concludes the paper and points some directions for future work.

\section{SCHEDULING AND DROP POLICIES}

To support different type of application with different type of scenario we have to schedule messages with their priority to destination node or intermediate nodes. First step, we are dividing that messages into three different classes which are ExpMsg (Expidted messages), NorMsg (Normal Messages), BulkMsg (Bulk messages). Then we send messages having different priorities with different routing algorithm, this technique is called as Priority+ scheduling policy. The flow of the priority+ scheduling algorithm is shown in following flowchart figure 3 . Another algorithm is about considering TTL and Prioirty of that particular message, The message which having high priority and high TTL value will schedule first. The flow of this algorithm is shown in figure 4 .

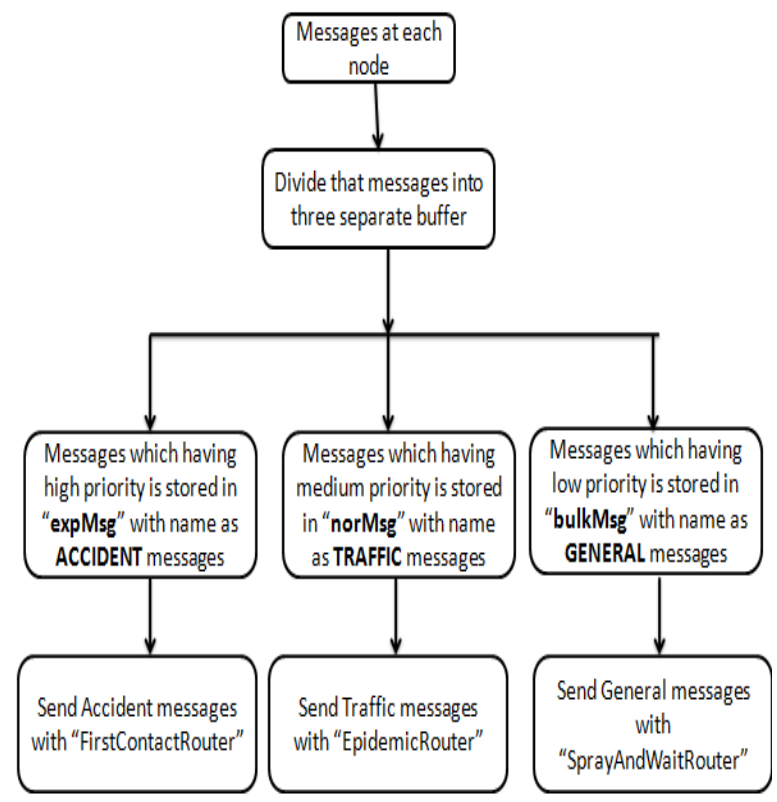

Fig 3: flow chart of PRIORITY+ scheduling policy

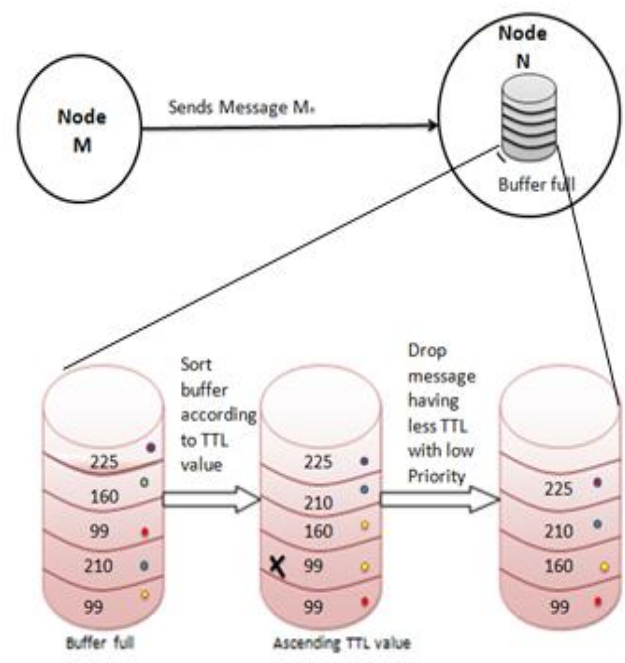

Fig4: FACTOR scheduling policy

\section{III.PERFORMANCE EVALUATION}

This section evaluates the impact of the above-described scheduling and drop policies on the performance of a previous define scheduling policy in DTN. For this purpose, a simulation study using the Opportunistic Network Environment (ONE) Simulator [10] has been executed. Next subsections present the simulation setup and results analysis.

Simualtion setup

In order to evaluate scenarios close to the reality, we use a real world map-based model of part of the Helsinki downtown area, with a dimension of $4500 \times 3400$ meters (Figure 3).

\begin{tabular}{|l|l|}
\hline Simulation time & $43200(12 \mathrm{Hrs})$ \\
\hline Interface Type & Bluetooth Interface \\
\hline Transmission speed & $2 \mathrm{Mbps}=250 \mathrm{kBps}$ \\
\hline Transmission range & $10 \mathrm{Meters}$ \\
\hline Mobile Node Speed & 2.5 to $4.0 \mathrm{~m} / \mathrm{s}$ \\
\hline SendQueueMode & $\begin{array}{l}\text { FIFO/RANDOM/ } \\
\text { PRIORITY+/FACTOR }\end{array}$ \\
\hline
\end{tabular}

Result Analysis:

Delivery probability defined as the number of packets sent from the sender and its successful reception at the destination.

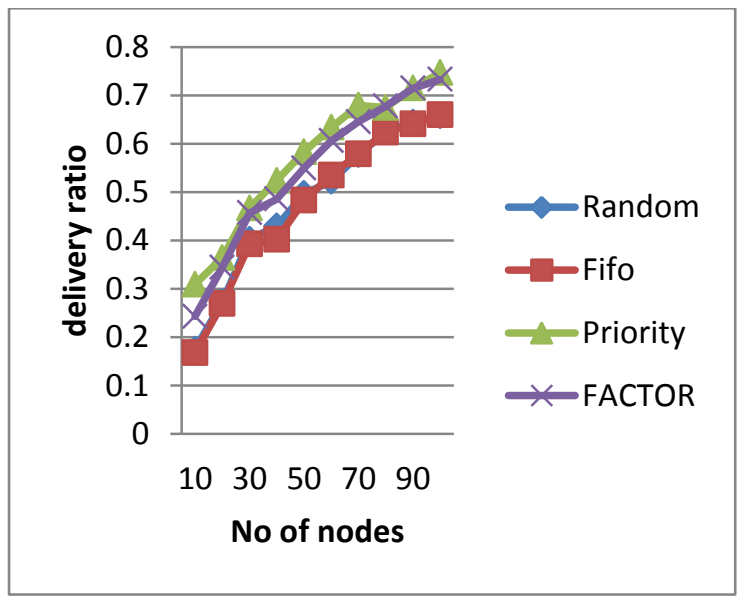

The overhead ratio is calculated using the following equation:

Overhead ratio $=($ Number of relaying messagesNumber of delivered messages)

Number of delivered messages

The latency measured here is the time that elapses between the creation of a message and its delivery at its destination. The average of the latency of the packets over the entire simulation time is considered.

This is the time as calculated for the delivered packets only. 

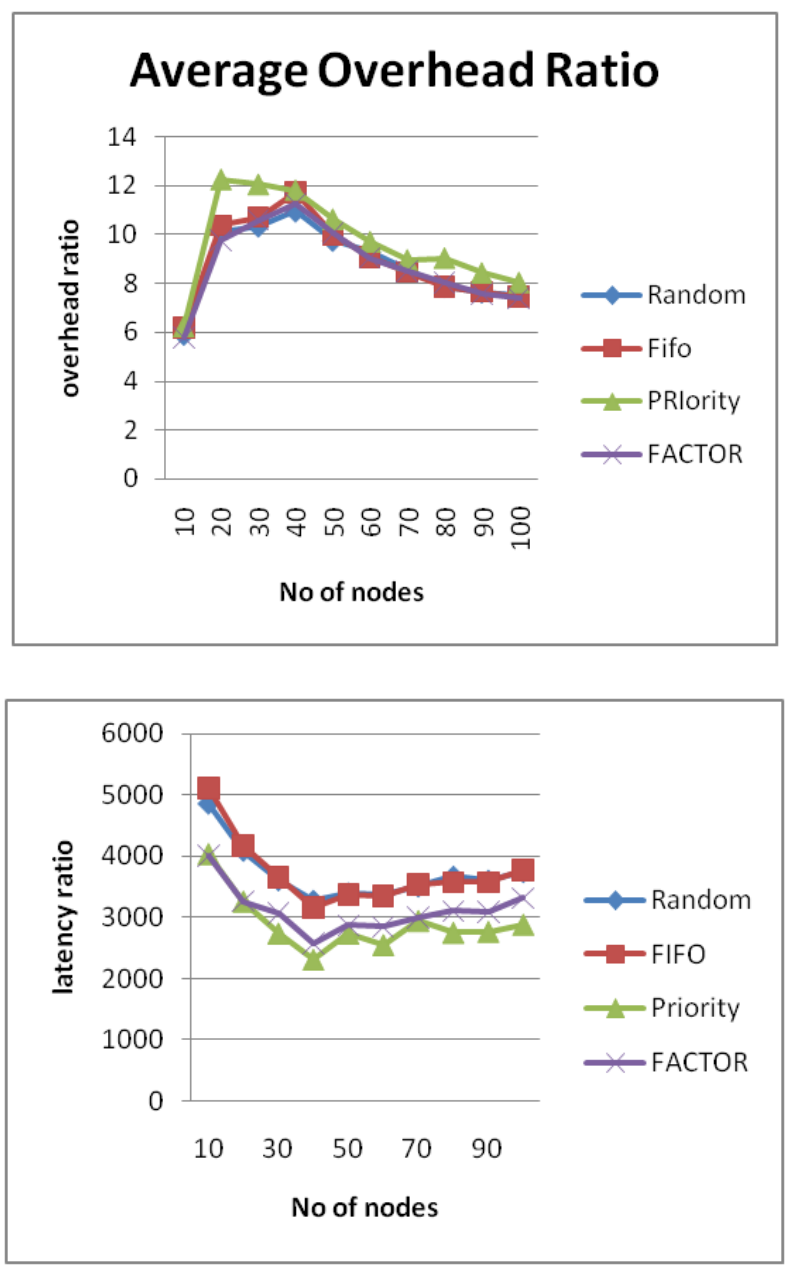

\section{IV.CONCLUSION}

In this paper, we investigated the problems of scheduling and buffer management in DTNs. We have proposed Priority+ and Factor scheduling technique using the ttl value of particular messages and their respective priorities. This gives around $10-15 \%$ more efficient result as compared to FIFO and random scheduling policies. We can send higher priority messages with higher ttl value first.

\section{REFERENCES}

[1] Kevin Fall, "A Delay-Tolerant Network Architecture for Challenged Internets" SIGCOMM'03, August 25-29, 2003.

[2] Padma Mundur, Matthew Seligman, "Delay Tolerant Network Routing: Beyond Epidemic Routing"IEEE 2008.

[3] K. Fall, "A Delay-Tolerant Network Architecture for Challenged Internet," in ACM SIGCOMM 2003, Karlsruhe, Germany, 2003, pp. $27-34$

[4] V. Cerf, S. Burleigh, A. Hooke, L. Torgerson, R. Durst, K. Scott, K. Fall, and H. Weiss, "Delay-Tolerant Networking Architecture," in RFC 4838, 2007.

[5] Xu Liu _and Yuanzhu Chen, "Report of A DTN Simulator - THE ONE", Department of Computer Science Memorial University of Newfoundland, May 11, 2013.

[6] Khalil Massri, Alessandro Vernata, Andrea Vitaletti, "Routing Protocols for Delay Tolerant Networks a Quantitative Evaluation", MSWiM'12, October 21-25, 2012, Paphos, Cyprus.

[7] http://www.netlab.tkk.fi/tutkimus/dtn/theone/.

[8] A. Demers, D. Greene, C. Hauser, W. Irish, J. Larson, S. Shenker, H. Sturgis, D. Swinehart, and D. Terry,"Epidemic Algorithms for Replicated Database Maintenance",Sixth Symposium on Principles of Distributed Computing, pages 1-12, August 1987.
[9] Michael Demmer, Eric Brewer, Kevin Fall, Sushant Jain, Melissa Ho, Robin Patra, "Implementing Delay Tolerant Networking", IRBTR-04-020, Dec.28 2004.

[10] Evan P.C. Jones, Paul A.S. Ward, "Routing Strategies for Delay Tolerant Networks", University of Waterloo 200 University Avenue West Waterloo, Ontario, Canada.

[11] A. Doria, M. Uden, and D. P. Pandey, "Providing connectivity to the saami nomadic community", 2nd International Conference on Open Collaborative Design for Sustainable Innovation (dyd 02), Bangalore, India, Dec 2002.

[12] G. E. Prescott, S. A. Smith, and K. Moe, "Real-time information system technology challenges for NASAs earth science enterprise", 20th IEEE Real-Time Systems Symposium, Phoenix, Arizona, Dec 1999.

[13] David (Bong Jun) Choi BBCR Lab., ECE, University of Waterloo, Supervisor: Professor Shen” Delay Tolerant Networks (DTNs): A Tutorial Challenges and Applications of Delay Tolerant Networks"

[14] Vasco N. G. J. Soares, Farid Farahmand, and Joel J. P. C. Rodrigues "'Scheduling and Drop Policies for Traffic Differentiation on Vehicular Delay-Tolerant Networks"

[15] João A. Dias, João N. Isento, Vasco N. G. J. Soares and Joel J. P. C. Rodrigues " Impact of Scheduling and Dropping Policies on the Performance of Vehicular Delay-Tolerant Networks" 978-161284-233-2/2011 IEEE

[16] Amir Krifa, Chadi Barakat and Thrasyvoulos Spyropoulos , "Message Drop and Scheduling in DTNs: Theory and Practice"'1536-1233/2012 IEEE

[17] Shou-Chih Lo, Min-Hua Chiang, Jhan- Hua Liou, Jhih-Siao Gao" Routing and Buffering Strategies in Delay-Tolerant Networks: Survey and Evaluation" National Dong Hwa University,2011

[18] Amir Krifa, Chadi Barakat" An Optimal Joint Scheduling and Drop Policy for Delay Tolerant Networks" 978-1-4244-2100-8/08, 2008 IEEE

[19] Vasco N. G. J. Soares, Joel J. P. C. Rodrigues, Paulo Salvador Ferreira, António M. D. Nogueira” Improvement of Messages Delivery Time on Vehicular Delay-Tolerant Networks"' 15302016/09 @ 2009 IEEE

[20] P. Juang, H. Oki, Y. Wang, M. Martonosi, L. S. Peh, and D. Rubenstein, "Energy-efficient computing for wildlife tracking: design tradeoffs and early experiences with zebranet", ACM ASPLOS, 2002. 